\title{
Upgrading ideas about the concept of Soft Computing*
}

\author{
Javier Montero \\ Faculty of Mathematics \\ Complutense University \\ 28040 Madrid, Spain \\ E-mail: javier_montero@mat.ucm.es
}

\author{
Luis Martínez \\ Department of Computer Science \\ University of Jaén \\ 23071 Jaén, Spain \\ E-mail:martin@ujaen.es \\ Received: 12-01-2010 \\ Accepted: 28-05-2010
}

\begin{abstract}
This short note is devoted to introduce the discussion carried out along this special issue on the concept of Soft Computing by key researchers in the field. We shall stress some aspects of the conception and origins of Soft Computing, supported by the scientific relevance of its participants. The contributors will show their own view about a single question, What is Soft Computing?, covering answers from a general historical approach to the role of some specific tools within their expertise. This discussion represents an extremely interesting view about the concept of Soft Computing, its meaning, its related techniques and its relationship with close fields.
\end{abstract}

Keywords: Artificial Intelligence, Complex Systems, Computational Intelligence, Computing with Words, Fuzzy Sets, Granular Computing, Machine Intelligence, Soft Computing, Soft Systems, Type-2 Fuzzy Sets.

\section{Introduction}

Soft Computing was introduced by Lotfi A. Zadeh $^{23,24,25}$ in the early 90's with the declared intention of covering a family of mathematical models and its related techniques that manage any kind of human uncertainty (including Probability, Fuzzy Logic and other linguistic-based models). But so far, there is no a clear and unique definition for Soft Computing that would be generally accepted. Such a lack of a clear definition for Soft Computing has soon addressed in the literature ${ }^{1,6,8,22,26}$. In particular, we can find several overlapped terminologies related to Soft Computing, like Soft Systems, Computing with Words,
Computational Intelligence, Machine Intelligence or Artificial Intelligence, and so on.

The fact is that during the last FLINS 2008 conference (Madrid, Spain, September 21-24, 2008) the plenary speaker Luis Magdalena ${ }^{10}$, Head of the European Centre for Soft Computing (Asturias, Spain), gave a talk under the title What is Soft Computing? that turned into an intense discussion among key researchers in the field attending Luis Magdalena's talk. The exchange of ideas was so vivid, complementary and illustrative, that we immediately contacted each active participant in such a discussion to write down their comments, in order to put them altogether as a special issue of some relevant scientific journal. Most of those FLINS participants agreed very soon to join this project,

\footnotetext{
* Work leading to this paper was partially supported by the Government of Spain, grants TIN2009-07901 and TIN2009-08286.
} 
and Da Ruan, founder of the FLINS initiative and that took also part in such a discussion, offered the International Journal of Computational Intelligence Systems for a rapid dissemination of such a wonderful and spontaneous round table, understanding that this project absolutely fitted the objectives of the journal and was within the best FLINS tradition. Some additional author was also invited to participate in this special issue due to their relevance in the topic and with the aim of keeping equilibrium of original positions in Luis Magdalena's session.

FLINS stands for Fuzzy Logic and Intelligent Technologies in Nuclear Science, an initiative launched by 1994 with the Belgian Nuclear Research Centre (SCK $\cdot \mathrm{CEN}$ ) with the objective to give $\mathrm{PhD}$ and Postdoc researchers the opportunity to carry out futureorientated research ${ }^{16}$. Since then, FLINS objectives have been extended to Computational Intelligence in Decision and Control, focusing on the promotion of research related to decision making and control problems for complex systems ${ }^{17}$. In fact, the declared main mission of FLINS is to conduct research on computational intelligence systems for solving intricate problems pertaining to nuclear/power research and related complex systems, bridging the gap between machine intelligence and complex systems via joint research with Belgian, European and international research institutes and universities. International FLINS conferences on computational intelligence systems encourage interdisciplinary research by bringing multidiscipline researchers together. The life discussion that naturally appeared around Luis Magdalena's talk was indeed in the best FLINS spirit and tradition.

\section{About this special issue}

This special issue has been conceived in three parts. Firstly, a general overview stated by Luis Magdalena ${ }^{11}$ is complemented with some historical and a crude computational blind analysis. The second part is centred on the complexity and the semantic component of natural intelligence. And the third part offers an alternative approach, focussing more on the sense of the question What is Soft Computing rather than the meaning of Soft Computing itself.

\subsection{Part 1: General Overview}

The first paper ${ }^{11}$ contained in this special issue is the extended version of the FLINS 2008 invited talk of Luis
Magdalena ${ }^{10}$, where the author states that there is no precise and generally accepted definition for Soft Computing. Magdalena briefly presents a first analysis of the main definitions of Soft Computing that can be found in the literature, developing a first comparative analysis, which leads him to postulate that there is no a significant difference between Soft Computing and Computational Intelligence, simply based on the relative presence of certain techniques and models.

In the second paper, Rudolf Seising ${ }^{20}$ offers a complementary historical perspective of the concept of Soft Computing, as introduced by Lotfi A. Zadeh, but linking his approach to the visionary calls of Warren Weaver and Charles Percy Snow towards a new interdisciplinary coming science associated to complexity and humanities, around fifty years ago.

From a crude spiking neural network approach, Liam Maguire ${ }^{12}$ then navigates across various overlapping terms like Computational Intelligence, Artificial Intelligence, Computer Science, Intelligent Systems, Intelligent Bio-inspired Systems or Computational Neurosciences.

\subsection{Part 2: Modelling Natural Intelligence}

The second part of the special issue starts with a paper of Enrique Ruspini ${ }^{19}$, who stresses the role of complexity of existing mathematical and computational models in the origin of Soft Computing, which is viewed as a strategy specifically devised to take advantage of the natural tolerance that imprecision and vagueness introduce in certain kind of problems. Enric Trillas, Claudio Moraga and Sergio Guadarrama ${ }^{21}$ then point out the underlying semantical-based Computing with Words challenge, studying the relationship between Soft Computing and Machine Intelligence in order to conclude that a Soft Computing implies a re-definition of traditional intelligence. Within this context, Witold Pedrycz ${ }^{15}$ focuses on Granular Computing, postulating that Type-2 Fuzzy Sets as an effective approach to deal with the complex linguistic structure that fuzzy sets requires for their required interpretability. And within the same context, Humberto Bustince ${ }^{3}$ stresses how those problems subject to a relatively precise solution make Interval Type-2 Fuzzy Sets a future main tool in Soft Computing. 


\subsection{Part 3: Complementary Views}

The third part of this special issue starts with a Janusz Kacprzyk's paper ${ }^{7}$ that from a discussion about the nature of intelligence, postulates that Soft Computing is a narrower area than Computational Intelligence. After considering the two aspects implied by the word Soft and the word Computing, this discussion will lead to relate Soft Computing to Soft Sciences and to enhance Checkland's Soft Systems Methodology as a well established approach to complex problem solving.

In addition, Piero Bonissone ${ }^{2}$ acknowledges the impossibility of a proper definition for Soft Computing, once he shows that such a semantic background suggests a continuously evolving model-builder.

Finally, Da Ruan ${ }^{18}$ presents a personal approach to the concept of Soft Computing based upon his practical experience in some particular extremely complex practical problems within SCK•CEN, pointing out the necessity of complementariness between new techniques and traditional approaches within realproblem solving.

\section{Final notes}

In this special issue key researchers in Soft Computing will enlighten and update some old concepts about Soft Computing, providing at the same time new paradigms and trends on the past and future of the field, in relation to close and certainly overlapping fields.

As promoters of this special issue, we want to stress the evident interdisciplinary characteristic of Soft Computing. Along this special issue, the authors make clear the deep connection established by Computer Scientists (including Computer Engineers and Mathematicians) to Social Sciences (including Management Sciences, Psychology and other fields where human expertise as decision makers is learnt) and Humanities (including Philosophy and Linguistic Sciences). Still, we should point out that one key piece is missing in the interdisciplinary view achieved in this special issue: Neurobiology, which is amazingly showing how the human brain builds up a decision making process by means of interactive but different machineries ${ }^{4,5,9}$. Computer scientists should devote more attention to the recent and expected findings in this field, in order to learn and create accurate models closer to the way natural intelligence approaches some decision making problems ${ }^{13,14}$.
We sincerely hope that the readers will enjoy this special issue.

\section{Acknowledgements}

We would like to thank Prof. Da Ruan, Editor-inChief of the International Journal of Computational Intelligence Systems, for his continuous support towards this project, and for accepting our demand to collaborate also as an author, in such a way this special issue can properly reproduce the ambient created around Prof. Magdalena's presentation, which for these two Guest Editors represents one of the most impressive scientific scenes ever. We of course must also thank the authors, their efforts to meet the required deadlines, specially to Prof. Lotfi A. Zadeh, prominent FLINS 2008 keynote speaker that due to an unexpected illness could not participate as a main author in this special issue, but has been so kind as to join the project through a suggesting foreword.

\section{References}

1. P. Bonissone, Soft Computing: the Convergence of Emerging Reasoning Technologies, Soft Computing 1, 618 (1997).

2. P. Bonissone, Soft Computing: a Continuously Evolving Concept, International Journal of Computational Intelligence Systems (this issue).

3. H. Bustince, Interval-valued Fuzzy Sets in Soft Computing, International Journal of Computational Intelligence Systems (this issue).

4. A. Bechara, H. Damasio, A.R. Damasio (2003). Role of the amygdala in decision making. Annals of the New York Academy of Sciences 985, 356-369 (2003).

5. A. Bechara, D. Tranel, H. Damásio, Characterization of the decision-making deficit of patients with ventromedial prefrontal cortex lesions. Brain 123, 2189-2202 (2000).

6. D. Dubois, H. Prade, Soft Computing, Fuzzy Logic and Artificial Intelligence, Soft Computing 2, 7-11 (1998)

7. J. Kacprzyck, Computational Intelligence and Soft Computing: Some Thoughts on Already Explored and not yet Explored Paths, International Journal of Computational Intelligence Systems (this issue).

8. X. Li, D. Ruan and A.J. van der Wal, Discussion on Soft Computing at FLINS'96, International Journal of Intelligence Systems 13, 287-300 (1998).

9. J.W. Kable, P.W. Glimcher, The neurobiology of decision: consensus and controversy. Neuron 63, 733745 (2009).

10. L. Magdalena, What is Soft Computing? Revisiting Arguments, Proceedings of the FLINS conference, 3-11 (2008). 
11. L. Magdalena, What is Soft Computing?, International Journal of Computational Intelligence Systems (this issue).

12. L. Maguire, Does Soft Computing Classify Research in Spiking Neural Networks?, International Journal of Computational Intelligence Systems (this issue).

13. J. Montero, Fuzzy Logic and Science, Studies in Fuzziness and Soft Computing 243, 67-78 (2009).

14. J. Montero, V. López, D. Gómez, The role of fuzziness in decision making. Studies in Fuzziness and Soft Computing 215, 337-349 (2007).

15. W. Pedrycz, Hierarchical Architectures of Fuzzy Models: from Type-1 Fuzzy Sets to Information Granules of Higher Type, International Journal of Computational Intelligence Systems (this issue).

16. D. Ruan, P D'hondt, P. Govaerts and E.E. Kerre, Eds. Fuzzy Logic and Intelligent Technologies in Nuclear Science. Proceedings International FLINS Conference, World Scientific (1994).

17. D. Ruan, J. Montero, J. Liu, L. Martínez, P D'hondt, and E.E. Kerre, Eds. Computational Intelligence in Decision and Control. Proceedings $8^{\text {th }}$ International FLINS Conference, World Scientific (2008).

18. D. Ruan, Lessons Learned from Soft Computing Applications at $S C K \cdot C E N$, International Journal of Computational Intelligence Systems (this issue).

19. E. Ruspini, Soft Computing and the Need to Cope with Complexity, International Journal of Computational Intelligence Systems (this issue).

20. R. Seising, What is Soft Computing? Bridging Gaps for $21^{\text {st }}$ Century Science, International Journal of Computational Intelligence Systems (this issue).

21. E. Trillas, C. Moraga, S. Guadarrama, A (naïve) glance at Soft Computing, International Journal of Computational Intelligence Systems (this issue).

22. J. L. Verdegay, R. Yager, P. Bonissone, On Heuristics as a Fundamental Constituent of Soft Computing, Fuzzy Sets and Systems, 159, 846-855 (2008).

23. L.A. Zadeh, Soft Computing and Fuzzy logic, IEEE Software 11, 48-56 (1994).

24. L.A. Zadeh, Fuzzy logic, Neural Networks and Soft Computing, Communications of the ACM 37, 77-84 (1994).

25. L.A. Zadeh, Fuzzy logic and Soft Computing: Issues, Contentions and Perspectives, Proceedings International Conference on Fuzzy Logic, Neural Nets and Soft Computing, 1-2 (Iizuka, Japan, 19994).

26. L.A. Zadeh, Applied Soft Computing, Applied Soft Computing 1, 1-2 (2001). 